\title{
Laboreal
}

Volume 15 NN$^{\circ} 2$ | 2019

Varia

\section{La psicología cubana y la salud de los trabajadores : entre el malestar y el bienestar}

A psicologia cubana e a saúde dos trabalhadores : entre o mal-estar e o bemestar

La psychologie cubaine et la santé des travailleurs : entre le mal-être et le bienêtre

Cuban psychology and workers' health : between malaise and welfare

\section{Jorge Juan Román Hernández}

\section{(2) OpenEdition}

Journals

Edición electrónica

URL: http://journals.openedition.org/laboreal/15521

DOI: $10.4000 /$ laboreal. 15521

ISSN: 1646-5237

\section{Editor}

Universidade do Porto

\section{Referencia electrónica}

Jorge Juan Román Hernández, «La psicología cubana y la salud de los trabajadores : entre el malestar y el bienestar », Laboreal [En línea], Volume 15 №2 | 2019, Publicado el 01 diciembre 2019, consultado el 11 abril 2020. URL : http://journals.openedition.org/laboreal/15521 ; DOI : https:// doi.org/10.4000/laboreal.15521

Este documento fue generado automáticamente el 11 abril 2020. 


\title{
La psicología cubana y la salud de los trabajadores : entre el malestar y el bienestar
}

\author{
A psicologia cubana e a saúde dos trabalhadores : entre o mal-estar e o bem- \\ estar \\ La psychologie cubaine et la santé des travailleurs : entre le mal-être et le bien- \\ être \\ Cuban psychology and workers' health : between malaise and welfare
}

Jorge Juan Román Hernández

\section{NOTA DEL EDITOR}

Manuscrito recibido en julio/2019

Aceptado tras peritaje octubre/2019

\section{Introducción}

1 En octubre de 2018, los profesionales cubanos de la salud ocupacional, particularmente los psicólogos, tuvimos el privilegio de recibir a numerosos colegas europeos, fundamentalmente franceses. La iniciativa de la profesora Dominique Lhuilier, del Conservatoire National des Arts et Métiers (CNAM) de París, de organizar un simposio en La Habana sobre la subjetividad y la salud de los trabajadores, desbordó toda previsión sobre sus positivos resultados. Las barreras lingüísticas no fueron óbice para la productividad de los intercambios entre los asistentes. Dejo constancia de nuestro profundo y sincero agradecimiento a la profesora Lhuilier por su elección de la sede del encuentro, gesto que nos honra y compromete. 
2 En mi intervención en el simposio pretendí expresar mis criterios sobre la situación actual y las perspectivas de desarrollo de la psicología aplicada a los problemas de la salud de los trabajadores en Cuba. Sin aspiraciones de exhaustividad, consideré imprescindible desarrollar en extenso condiciones sociales, socio-laborales, de la salud ocupacional, así como de la ciencia psicológica en el país. Esas condiciones configuran contextos que influyen en la elaboración de la conducta del sujeto en ámbitos laborales y extralaborales (Román, 2017a, 2017b, 2019).

\section{Escenario socio-laboral cubano}

\subsection{El proyecto de sociedad}

3 Cuba es un país económicamente subdesarrollado en el cual rige un sistema social socialista, oficialmente proclamado en 1961, cuyo fundamento ideológico es el marxismo-leninismo. Los medios fundamentales de producción son propiedad del Estado. La economía se basa en la planificación estatal. La estructura de gobierno y de administración es estratificada y altamente centralizada.

4 En términos poblacionales, según el Anuario estadístico de Cuba (Oficina Nacional de Estadística e Información, 2019), al término del año 2018, existían 11.209628 habitantes. De esos, 7146900 (63.8\%) se encontraban en edad laboral, y estaban ocupados 4.482700 (39.9\%). De los ocupados, 3.067000 (68.4\%) eran del sector estatal, definitivamente mayoritario. La tasa de desocupación en el propio año fue de $1.7 \%$.

5 Tras la toma del poder político en 1959, se adoptaron medidas conducentes a extinguir las diferencias de clases. Dos leyes de reforma agraria suprimieron los latifundios, que fueron parcelados y su propiedad pasó a los campesinos que laboraban en esas tierras, o se dedicaron a granjas estatales. Se nacionalizaron las empresas de mayor significación económica, varias de capital extranjero. En 1968, la llamada Ofensiva revolucionaria incrementó el poder del Estado sobre todos los medios de producción y las empresas de servicios, incluidas actividades privadas menores.

6 La burguesía nacional fue despojada de su poder económico y desapareció como clase social fundamental. Las empresas norteamericanas fueron las más perjudicadas con las expropiaciones. Se generó una larga historia de desavenencias y confrontaciones que resiste el paso del tiempo.

7 Se tomaron medidas para nivelar las diferencias económicas en la población, sin dejar de considerar el principio de retribuir según el trabajo. Sin embargo, no dejaron de existir diferencias sociales en la esfera del consumo.

En la actualidad, las diferencias sugieren no la existencia de clases propiamente dichas, porque el Estado se reserva la propiedad de los medios de producción fundamentales. Sin ser su única fuente, esas diferencias también se nutren de irregularidades en la esfera de la distribución y por tanto, del consumo.

9 La Constitución, aprobada por referendo el año 2018, en su artículo 1 caracteriza al Estado cubano como un Estado "de trabajadores". En el artículo 3 afirma que todo el poder del Estado dimana de la soberanía del pueblo. Sin embargo, el partido comunista es definido en el artículo 5 como la "fuerza dirigente superior de la sociedad y del Estado" (Constitución de la República de Cuba, 2019). En consecuencia, el silogismo 
coloca a la organización política como intérprete y modeladora de la soberanía nacional. No existe otro partido político oficialmente reconocido.

La salud y la educación son gratuitas. La propiedad de la vivienda fue entregada a sus moradores. Se proporcionan las condiciones para el empleo pleno, y se prohíbe todo tipo de discriminación.

11 En el texto constitucional se preservan otros derechos civiles (igualdad, creencia, conciencia, palabra, expresión, reunión, asociación, manifestación, opinión, etc.) ; cuyo disfrute -explícitamente se reitera- no puede contraponerse al orden social establecido. Más aún, en el artículo 3 se legitima el derecho ciudadano de "combatir por todos los medios" a cualquiera que intente derribar el orden político, social y económico.

Los sindicatos, como las demás organizaciones sociales, que Lenin llamó "poleas de transmisión", tienen la función de transmitir la política del partido y convocar a los trabajadores para cumplirla. Asumen la representación de los trabajadores y median en la solución de conflictos con las administraciones de las instituciones laborales. Los sindicatos se subordinan a una instancia superior centralizada. No se admite la existencia de sindicatos autónomos.

La identificación ideológica y política del partido, del Estado y de las organizaciones sociales, entre las que se incluyen los sindicatos, se garantiza además porque la pertenencia al partido es un criterio de peso que se toma en cuenta para ocupar cargos directivos en cualquier nivel.

Las fuentes principales de ingreso de divisas del país son la exportación de servicios técnicos, fundamentalmente relacionados con la salud y el turismo. El sistema de dirección y gestión empresarial actual, conocido como "perfeccionamiento empresarial", se caracteriza por preservar y favorecer la centralización.

Los trabajadores tienen el derecho de manifestar directamente sus criterios, para lo cual se convocan asambleas periódicas de conjunto con los directivos de las instituciones laborales. Existen modos indirectos, a través de sus representantes en órganos de dirección, por ejemplo. Los cambios de importancia en la legislación laboral son motivo de análisis y propuestas en asambleas de trabajadores convocadas a esos fines.

17 No hay sociedad en que sus ciudadanos, de modo homogéneo, sigan al pie de la letra todas sus normas. Tampoco existe un régimen social que carezca de una base de apoyo en la sociedad que lo legitime y sostenga.

\subsection{El proyecto en la práctica}

El proyecto social, que tiene más de 60 años de existencia, si partimos de la toma del poder en 1959, se encuentra expuesto a dificultades derivadas de la acción de factores externos e internos.

En el caso cubano, los factores externos más relevantes que han afectado a la sociedad son el bloqueo o embargo económico y comercial impuesto por los Estados Unidos y, por otra parte, la desaparición del campo socialista y de la otrora Unión Soviética. 
20 La confrontación entre los gobiernos de Estados Unidos y Cuba ha llegado a extremos de poner en peligro la paz mundial. El embargo eleva los costos de productos de diverso grado de necesidad, entre los cuales, tecnologías y medicamentos de última generación.

21 La desaparición del campo socialista en el este de Europa y la Unión Soviética, sumió al país en una grave crisis económica, dado que en gran medida sus finanzas dependían de esas relaciones.

22 No es posible atribuir enteramente a razones externas o internas la actual situación de la sociedad cubana. El mensaje político oficial con frecuencia es francamente externalista, lo cual no es enteramente satisfactorio porque puede ofrecer una imagen parcializada de relativa credibilidad, y se desvía la atención de las condiciones sobre las que se puede tener mayor influencia.

23 La resistencia a los cambios que demanda la realidad social y que favorecerían la dinamización de la economía y una mejora de las condiciones de vida de los ciudadanos es una manifestación del peso que tiene la subjetividad de la alta dirigencia del país, o al menos de una parte de quienes la componen.

24 La ineficiencia de la economía no constituye secreto alguno. El Producto interno bruto en 2018 fue de 1,4\%. El más elevado desde el año 2012 fue de 4,4\% en 2015. Comparándolo con el PIB promedio de América Latina de 1,2 \%, el ministro de economía calificó el de Cuba como no suficiente, pero "meritorio" (Figueredo \& Concepción, 2018).

El trabajo autónomo fue autorizado en 2005. No pocas personas entrevistadas por el autor en la consulta clínica, paradójicamente preferían perder el empleo para dedicarse a una actividad privada, con la que aspiraban liberar su capacidad e iniciativa, mejorar su calidad de vida y la de su familia.

Educación y salud son dos sectores priorizados a los que se ha dedicado el $51 \%$ del presupuesto nacional en 2019 ; pero no por ello son inmunes al contexto descrito. La exportación masiva de servicios técnicos, con amplia mayoría de los de salud, constituye la mayor fuente de ingreso del país.

Se ha procurado mantener los servicios básicos, e incrementar los ingresos de la población, con vista a proteger a quienes se encuentran en mayor desventaja; o estimular a quienes trabajan en sectores prioritarios, como son la salud y la educación ; pero no por ello superan sus necesidades (Molina, 2016).

Desde 1993 existen dos monedas, una de las cuales es convertible en divisa extranjera con una relación de cambio de $1: 24$ con la moneda nacional. Si en sus inicios la medida fue útil, en la actualidad es un problema complejo pendiente de solución. Basta aclarar que como los salarios se pagan en la moneda nacional y no todas las personas tienen acceso directo a la convertible, en la que se fijan los precios de no pocos productos necesarios ; aunque se admite el pago en la moneda nacional respetando la relación de cambio antes apuntada.

Según la Oficina Nacional de Estadística e Información (ONEI) (2019) el salario medio en 2018 fue de 777 pesos, moneda nacional.

Factores como el trabajo privado, la existencia de doble moneda, los apoyos familiares desde el exterior, las gratuidades existentes (vivienda, salud, educación), a lo largo de los años han polarizado económicamente a la población y por tanto también en su nivel y calidad de vida. 
31 Demográficamente, dos fenómenos son característicos: los flujos migratorios y el envejecimiento poblacional. Los primeros suceden hacia el exterior del país, y de zonas rurales a las ciudades. Estos han aumentado entre los sectores más jóvenes. El envejecimiento plantea demandas específicas de diverso tipo: constructivas, alimentarias, de servicios, ocio, y a la vez disminuirá la fuerza de trabajo disponible.

\subsection{Las consecuencias}

Antes de centrar la atención en dificultades, limitaciones y desaciertos, es preciso aclarar que no faltan consecuencias positivas. Entre ellas han prevalecido el sentido de identidad, que, pese a las desavenencias y el alejamiento, ha mantenido vivo el espíritu y la cultura de la nación. Se añaden la solidaridad, el sentido de responsabilidad por la familia, la ingeniosidad y creatividad sobradamente probadas en tiempos difíciles.

La actualidad nos presenta un panorama muy complejo que enfrenta la población cotidianamente, en particular los trabajadores (el orden de mención no expresa prioridad) :

- La emigración y el envejecimiento disminuyen la fuerza de trabajo.

- Profesionales experimentados han abandonado su trabajo para dedicarse a una ocupación menos calificada, pero mejor retribuida.

- Los incrementos compensatorios de salarios y pensiones no alcanzan proporciones comparables al que ha tenido el aumento del costo de la vida.

- La oferta de productos alimenticios en un mercado normado a precios subsidiados no alcanza a cubrir las necesidades de la población y los precios de oferta y demanda en cualquier moneda, son elevados.

- La ocurrencia de hechos de corrupción y delictivos ha sido favorecida por las dificultades económicas, y el bajo control de los bienes.

- La convivencia cotidiana con la irregularidad, la transgresión de la ley y hasta la corrupción, contradictoriamente son percibidos por la población con naturalidad y resignación.

- Desvalorización del trabajo. Los ciudadanos, en particular los jóvenes, manifiestan escepticismo sobre el valor del trabajo como medio de crecimiento personal y de progreso en la escala social.

- Medidas para motivar el trabajo (reconocimientos morales, incrementos salariales, contrataciones de jubilados, incentivos materiales de bajo costo), antes efectivas, carecen de suficiente valor de estímulo actualmente.

- La elevada cautela y dilación en la toma de decisiones refleja resistencia al cambio.

- No ha sido posible diseñar un sistema de transporte estable, eficiente y sostenible que garantice, en particular, la movilidad de los trabajadores.

- La construcción de viviendas continúa siendo un problema no resuelto.

- La calidad de la enseñanza ha mostrado debilidades organizativas que afectan su misión social.

- Los servicios de salud también se han resentido por la falta de recursos y medicamentos

- El trabajo expatriado es "tercerizado" por una agencia empleadora estatal. El trabajador se favorece económicamente; pero como viajan sin sus familiares, la ausencia prolongada puede dar lugar a la falta de influencia en los hijos, sobrecarga doméstica de la pareja y hasta fractura familiar.

- En el caso particular del sector de la salud, el ministerio correspondiente es el único empleador de médicos, tecnólogos de la salud y enfermeros, lo que da lugar a una relación 
de poder desventajosa para el trabajador, quien pudiera limitarse en la expresión de sus discrepancias o iniciativas. cuales los trabajadores. Estas son fuente de tensiones emocionales que se agregan a las generadas por otras circunstancias de la vida. La elaboración del síntoma no es infrecuente que conduzca a estados más complejos, como la depresión, por ejemplo. La demanda de servicios de salud mental no ha conducido hasta el momento al incremento de la formación de especialistas, a pesar de que los servicios de psiquiatría han ocupado durante años el $3^{\circ}$ o $4^{\circ}$ lugar en la cantidad de consultas efectuadas por especialidad (Román, 2017a).

Ciertas conductas que se observan en predios y colectivos laborales, sobre todo los estatales, pueden interpretarse como respuestas defensivas compensatorias de la carga laboral y extra-laboral cotidianas. Pueden tener diverso grado de conciencia a pesar de que sean actitudes de potencial generalización en los colectivos laborales. Su reiteración y expansión intencionada, su comisión en colectivo y en condiciones de real desgaste por el trabajo, puede merecer que sean consideradas como verdaderas ideologías defensivas en el concepto formulado por Dejours (1990). Entre ellas, las dos siguientes :

- Graduación subjetiva del esfuerzo en concordancia con la estimación de la retribución ("hacen como que me pagan y hago como que trabajo").

- Desvalorización del sentido y objetivos del trabajo dando prioridad al cumplimiento formal del rol (por ejemplo, enfocarse no en las necesidades de un paciente o un cliente, sino en las acciones que debe realizar).

La autoridad administrativa y la moral de trabajo pueden disminuir por efecto de condiciones que afectan a todos, directivos y dirigidos. Se incrementa la tolerancia a lo irregular. A veces al punto de consentir modificaciones en la organización del trabajo que afectan, o al menos potencialmente pueden afectar el cumplimiento del mismo.

La desvalorización es capaz de transferirse de un ámbito a otro, o transmitirse de una persona a otra, generalizarse. Su antídoto no puede limitarse a apelativos morales e ideológicos, que no guarden significación para el sujeto.

La existencia de una "delincuencia organizacional", como la llama Barral (2010), constituye un caso extremo, aunque no infrecuente. Sobre la base de años de investigación, este autor la caracteriza del siguiente modo :

- Conjunto de relaciones institucionales que incluye a personas de diverso nivel jerárquico.

- Involucra, preferentemente a trabajadores de las áreas de mayor ganancia potencial fraudulentas.

- La ganancia fraudulenta se obtiene por diversas vías: alteración de los documentos financieros, de los inventarios, liquidación de los planes económicos en valores para apropiarse de los excedentes, alteraciones de los precios de venta públicos, gestiones individuales normalmente engorrosas y dilatadas, tráfico de influencias y favores, oportunidades de publicitarse y promocionarse a través de los medios de comunicación, entre otras.

- Es un antisistema social producto de una dinámica particular de las relaciones económicas que modifica las relaciones sociológicas $\mathrm{y}$, como consecuencia, genera problemas ideológicos. 
- En las organizaciones laborales afectadas, bajo la institución formal, aparece una más real institución sumergida.

Esto último resulta de gran interés, pues representa no la apropiación de una producción dada que configuraría un robo ordinario ; sino el usufructo de los medios de producción, en cierto modo una apropiación temporal de los mismos para beneficio propio. Los productos se destinan no solamente al mercado negro; sino también al comercio en establecimientos de venta pública, generalmente estatales.

Simultáneamente, van modificándose los valores de la población o de una parte de ella, asumiendo con relativa tolerancia la existencia y la inevitabilidad de estos hechos. El lenguaje popular lo ilustra : el concepto de "robar" cambia al más concesivo y ambiguo de "luchar".

La base social de apoyo del sistema se enturbia. No solo la conforman quienes, con real integridad ideológica sinceramente creen en el mismo ; sino también quienes medran en irregularidades como las antes descritas y prefieren su perpetuidad. La existencia de ese sector oportunista de apoyo, resulta difícil de identificar.

\section{La psicología en el contexto de la salud ocupacional}

2 La salud ocupacional tuvo una magra representación en los contextos laborales antes del período revolucionario. Existían ordenanzas que establecían las inspecciones sanitarias estatales, así como chequeos médicos periódicos en trabajos con riesgos laborales identificados.

\subsection{Tiempos de auge}

\section{Período 1978 - 1985}

La salud ocupacional tuvo un pujante desarrollo en el país después de 1959. Más de dos mil médicos fueron ubicados en centros laborales, se creó una red de centros provinciales y municipales con departamentos dedicados a la salud ocupacional, y se logró una cobertura nacional. En 1978 se fundó el Instituto de Medicina del Trabajo (IMT) por el profesor Antonio Granda Ibarra. Le acompañaba un psicólogo del trabajo recién graduado, Pedro Almirall Hernández que inició investigaciones sobre carga mental, y neurotoxicología (Almirall, 2001, Granda, 1992).

La existencia de una residencia médica en Medicina del trabajo formaba a los especialistas necesarios para desarrollar la salud ocupacional. Varios cursaron estudios en países de Europa del este.

La Ley 13 de 1977 (Asamblea Nacional del Poder Popular, 1977) rigió la salud y seguridad de los trabajadores, fortaleció la inspección sanitaria estatal y cumplió una función metodológica, con rigurosas regulaciones. Importante fue la colaboración con instituciones homólogas de la Unión Soviética y de los países socialistas de Europa del este, bajo la coordinación del multinacional Consejo de Ayuda Mutua Económica (CAME). Su programa de ciencia y técnica contenía una línea de investigación sobre la higiene del trabajo y enfermedades profesionales.

Desde mediados de los años 80 este autor gestionó por la parte cubana el plan de colaboración científica en salud ocupacional del CAME. Participaban Alemania, 
Bulgaria, Checoslovaquia, Hungría, Polonia y la Unión Soviética. Solamente Alemania, Hungría y Cuba trabajaban en investigaciones psicológicas. En los dos primeros países, los trabajos eran más bien dedicados a investigaciones de carga mental, psicofisiología, neurotoxicología, condiciones y ambiente laboral.

A pesar del llamado en 1974 de la $27^{a}$ Asamblea Mundial de la Organización Mundial de la Salud (OMS) (Organización Mundial de la Salud, 1974) sobre la necesidad de dedicar esfuerzos investigativos a las condiciones sociales del trabajo y su relación con la salud, diez años después solo Cuba entre los países del CAME tenía una línea de investigación sobre ese tema. La historia posterior no permitió establecer una colaboración.

Fueron años dedicados a la organización e inicio del trabajo de investigaciones y clínico. Se realizaron también numerosos servicios a empresas de evaluación de riesgos diversos y chequeos médicos a trabajadores.

51 Las investigaciones se fueron agrupando en líneas específicas. Se iniciaron nuevas actividades docentes como la Maestría Internacional de Salud Ocupacional. Se procuraba dar visibilidad al trabajo a través de publicaciones en el ámbito nacional, capacitaciones, divulgación, servicios a empresas, etc.

Período 1985 - 1991

53 A pesar del cierre de la especialidad médica de Medicina del Trabajo, la salud ocupacional y la psicología a ella aplicada, experimentaron tiempos de auge.

En la salud ocupacional, se amplió la cobertura de sus servicios a todo el país, y se firmaron convenios para la formación posgraduada de investigadores en países socialistas, se estableció un plan de investigaciones y se incrementaron los recursos materiales.

En la psicología, a pesar de lo reducido del grupo, pues se contaba con cinco investigadores e igual número de auxiliares, hubo cambios favorables de diverso tipo: se creó un departamento independiente, el índice de publicaciones por investigador como promedio fue superior al de los restantes colectivos y se editó un Manual de evaluación de trabajadores expuestos a neurotóxicos (Almirall, Mayor, Del Castillo, Rodríguez \& Román, 1987). Tres psicólogos alcanzaron su doctorado. El tema psicosocial, que hasta entonces se había limitado a la estimación subjetiva de la carga de trabajo, fue adquiriendo progresivamente mayor elaboración teórica, con el referente de la teoría de la alienación de Marx y sus posteriores desarrollos. Se incrementaron las investigaciones empíricas en equipos multidisciplinarios conformados por investigadores de diversas disciplinas mayoritariamente del sector de la salud. Se definieron como líneas de investigación fundamentales : neurotoxicidad, factores psicosociales, estrés, riesgos físicos, salud mental y trabajo. Prevalecieron las investigaciones de carácter cuantitativo.

Esas líneas requieren de una organización multidisciplinaria del trabajo de investigación. Tal organización se evidencia en los autores de los artículos publicados en la Revista Cubana de Salud y Trabajo (www.revsaludtrabajo.sld.cu/index.php/ revsyt).

En relación con el estudio del trabajo, existieron tres institutos de investigación. Uno, dependiente del Ministerio del Trabajo abordaba temas de seguridad y protección del trabajo ; otro, llamado de Investigaciones Científicas del Trabajo abordaba temas de organización del trabajo, economía del trabajo y estudios ergonómicos. Ambos dejaron de existir hace años. Los temas de salud son asumidos por profesionales del actual 
Instituto Nacional de Salud de los Trabajadores (INSAT), e instituciones de salud, universidades y empresas, algunas, del interior del país.

La Maestría en Salud Ocupacional iniciada en este período, incluía un módulo de psicología. Además, se impartieron capacitaciones en instituciones laborales. Se realizaron servicios de evaluación psicológica en empresas y una consulta clínica. Estudiantes universitarios realizaban pasantías.

La salud ocupacional vivió una época de auge y proporcionó un área de trabajo inédita para la ciencia psicológica en Cuba.

\subsection{Tiempos de crisis} salud ocupacional. Abruptamente, planes de colaboración en investigaciones, formación posgraduada e intercambio de especialistas, perdieron toda efectividad. Disminuyeron los recursos para el trabajo, equipamiento, reactivos, entre otros. Medidas generales afectaron también el trabajo, como largas interrupciones del servicio eléctrico, falta de transporte público, disponibilidad de alimentos. Se cerraron numerosos centros laborales y en otros se disminuyó la jornada laboral o los días de presencia. dadas las limitaciones objetivas. En particular, las carencias bibliográficas se acrecentaron, dificultando sobremanera la actualización de la información. epidemiología. Las expectativas de que una parte de los graduados de la residencia en higiene y epidemiología se dedicara a la medicina del trabajo, ya fue evidente en estos años que no se cumpliría en la práctica. Desde entonces no se ha graduado un especialista más. Los egresados de la Maestría tampoco han satisfecho esa necesidad.

A los fines de reforzar la atención médica en la comunidad, se fundó la especialidad de Medicina General Integral, con énfasis en pediatría, obstetricia, clínica y psicología. Sus egresados fueron denominados médicos de familia, parte de los cuales se destinaban a centros laborales.

Sin embargo, luego se redujo la ubicación de médicos de familia en centros laborales. En el año 1995 llegaron a la cantidad de 2304, en 2002 quedaban 876, y actualmente ya no se reportan datos (Ministerio de Salud Pública, 1986-2002).

Entre 1988 y 2018, la cifra más alta de médicos del trabajo se alcanzó en 1989 con 375 médicos, de los cuales 181 especialistas titulados y 194 "dedicados", quienes, sin poseer el título de especialista, por su experiencia se habilitan para ejercer como médicos del trabajo. Al no haber nuevas graduaciones, la cifra anterior fue reduciéndose por diversas causas: fallecimientos, emigración, traslado de institución, etc. Según los datos oficiales, en la actualidad existen en todo el país para atender la salud laboral de más de 4 millones de trabajadores solamente 104 médicos, de los cuales solo 55 son especialistas titulados en medicina del trabajo (Ministerio de Salud Pública, 1995-2018). 

Dado que progresivamente disminuyen los especialistas por razones diversas (jubilación, emigración, fallecimiento), y que en las instituciones de salud rige la norma, integrada a la cultura de la organización, de que las instituciones de salud en lo posible deben ser dirigidas por médicos, es razonable albergar inquietudes sobre la supervivencia de la especialidad y de las restantes disciplinas afines, entre las cuales, la psicología.

74 La inspección sanitaria estatal no se ha recuperado. La cobertura nacional de los servicios de salud ocupacional no se vislumbra como una posibilidad real. La ley 13 fue derogada y en su lugar ha quedado el Código de trabajo (Asamblea Nacional del Poder Popular, 2014) cuyo contenido no posee la especificidad necesaria y el detalle de la ley suplantada.

75 Una consulta anónima a once expertos en salud ocupacional de diverso perfil profesional fue realizada para conocer su valoración del estado actual de la salud ocupacional. Los requisitos de su elección fueron tener más de 15 años de trabajo en salud ocupacional, no tener responsabilidades que permitieran la toma de decisiones estratégicas, y su voluntaria participación. Las evaluaciones más bajas las recibieron la formación de pregrado, y la efectividad del liderazgo de los decisores. Las más favorables recayeron en el nivel científico de los profesionales y técnicos en ejercicio, y la visibilidad internacional de la Salud ocupacional cubana, por cierto, considerada mayor que la visibilidad nacional (Román, 2017b).

Las perspectivas de desarrollo de la psicología se cifran no solo en los ya dedicados a la salud ocupacional, sino también en otros profesionales de la salud, docentes universitarios, psicólogos de empresas que han incorporado contenidos de salud ocupacional a sus trabajos, por ejemplo, véase Medina (2018). Las carencias bibliográficas no han aminorado sustancialmente debido a dificultades para adquirir suscripciones a publicaciones periódicas. El servicio Hinari de la OMS que permitía el libre acceso a cientos de revistas, entre las cuales de salud ocupacional y de psicología, retiró la gratuidad y cerró el acceso a la mayor parte de sus fondos. Durante años, el acceso a Internet estuvo vedado.

77 Otro ejemplo del interés por este campo es la proporción de artículos con participación autoral de psicólogos de diversa afiliación institucional en la Revista Cubana de Salud y 
Trabajo. Contabilizados los datos desde su primer número en el año 2000 hasta 2019, se han editado 45 números, con un total de 400 artículos, de los cuales más de la cuarta parte de sus autores $(26,5 \%)$ son psicólogos.

En el año 2018, se indicó a la dirección del INSAT reestructurar la institución mediante la fusión de algunos de sus componentes.

Contrariamente a lo esperado, el departamento de psicología fue fusionado con el de fisiología ; en una decisión inmerecida e impuesta sin la participación plena y previa de los afectados.

\section{El psicólogo de la salud ocupacional en Cuba}

En la década de los años 60 , entre los primeros psicólogos egresados con formación clínica, un pequeño grupo debió enfrentar diversos problemas no incluidos en sus programas docentes. Se trataba no solo de atender la clínica de los trastornos mentales, sino sobre todo colaborar en numerosos programas especiales de atención comunitaria (materno infantil, conducta suicida, tercera edad, cáncer, etc.), ninguno específicamente dedicado a la salud del trabajador. Sin embargo, las exigencias concretas de la práctica profesional se impusieron. Con más inspiración práctica que bagaje teórico, desarrollaron un campo de aplicación de la ciencia psicológica que denominaron "psicología de la salud", varios años antes de que la American Psychological Association la reconociera como una sub-disciplina en 1995.

Solemos distinguir entre el psicólogo del trabajo y el psicólogo de la salud ocupacional.

El estudio, la investigación y la práctica profesional en relación con la actividad laboral entraña una postura y un compromiso ético que encuentra su expresión en la actitud del investigador, independientemente de la especialización que practique.

La psicología del trabajo y la psicología organizacional surgieron con propósitos aún vigentes, que a la larga están dirigidos a incrementar la productividad y la calidad del trabajo; mejorar las condiciones de trabajo, las relaciones humanas, manejar los estímulos al trabajo, pero el foco fundamental está en el incremento de la eficiencia. No es un propósito ilegítimo. El trabajador necesita del trabajo y de la valoración de sus resultados; pero el hombre es percibido como recurso. Recurso que hay que preservar. El bienestar y la salud ocupan un segundo espacio. Algunos prescinden hasta de considerar esos asuntos.

Percibimos en cambio, a la psicología de la salud ocupacional de modo inverso. Su énfasis se coloca en la prevención y la promoción de la salud de los trabajadores y, secundariamente, con esas medidas de cuidados y protección de la fuerza laboral, favorecer el desempeño de sus tareas. Dejours (1990) refiere : "Lo que parece verdadero desde un punto de vista de la productividad es falso mirándolo desde la economía del cuerpo..." (p. 48).

Dos razones de carácter más práctico que conceptual. En primer lugar, la compartimentación de los objetos sociales de los organismos de salud por un lado, y de trabajo por el otro, sugieren la distinción que hacemos. En segundo lugar, en los programas universitarios de pregrado el más afín, de Psicología del trabajo y de las organizaciones laborales, no incluye contenidos de salud. 


\subsection{Realizaciones}

Las realizaciones se exponen de modo resumido en cuatro áreas de desempeño: investigación, docencia, atención clínica y servicios.

En la investigación se pueden mencionar los siguientes : Batería de pruebas cognitivas digitalizada para el diagnóstico de la acción de sustancias neurotóxicas; Ergonomía cognitiva en el estudio de puestos de trabajos de altas demandas intelectuales; Complementación del modelo de R. Karasek sobre la tensión emocional mediante la adición de una dimensión motivacional; Sistema de vigilancia epidemiológica de riesgos psicosociales en la Fiscalía General de la Nación de Colombia ; Estrés psicosocial en gestantes trabajadoras; Adaptación de cuestionarios diversos para la evaluación psicológica en salud ocupacional

En Cuba, la formación posgraduada del psicólogo en contenidos de salud ocupacional, ha quedado a expensas de programas de postgrado que los incluyen con diferente amplitud y profundidad. Entre esos, la Maestría en salud ocupacional, del Instituto Nacional de Salud de los Trabajadores ; las maestrías en Salud Pública y en Psicología de la Salud, ambas de la Escuela Nacional de Salud Pública ; la Especialidad en Psicología de la Salud, de la Universidad de Ciencias Médicas de La Habana y la Maestría en Psicología del Trabajo y de las Organizaciones Laborales, de la Facultad de Psicología de La Habana, así como la Maestría en Salud y Seguridad del Trabajo, de la Facultad de Ingeniería Industrial de la Universidad de Ciencias Tecnológicas (Universidad de Ciencias Tecnológicas, 2016 ; Universidad de Ciencias Médicas de La Habana, 2016).

9 A solicitud de las empresas algunos temas son objeto de talleres y capacitaciones específicas.

Con la vista puesta en el futuro, se imparte un curso optativo de pregrado en la Licenciatura de Psicología.

1 La atención clínica, fundamentalmente diagnóstica, se realiza un día en la semana. Los estudios de casos favorecieron la experiencia profesional que nos permitió cobrar conciencia de las limitaciones de las técnicas cuantitativas. Los servicios a empresas básicamente son solicitados por las organizaciones laborales. Son sumamente diversos : sobre determinada condición peligrosa, de estrés, vigilancia, prevención de accidentes, etc. Sin embargo, la fascinación por el número, la proporción, las tasas, etc., siempre prevalece entre quienes solicitan los servicios, por lo cual es difícil dar la espalda totalmente a la cuantificación.

2 Los colectivos laborales son casi en su totalidad entusiastas y colaboradores. No resulta difícil obtener la mejor disposición y apoyo en las investigaciones de los trabajadores, directivos y sindicatos.

Resulta imposible relacionar la cantidad de publicaciones en artículos, capítulos de libros e informes realizados por los psicólogos cubanos en el campo de la salud ocupacional. Lo mismo sucede con la cantidad de cursos de postgrado impartidos y las contribuciones a eventos científicos, tanto en Cuba como en otros países.

A pesar de la crisis económica, y muy probablemente a consecuencia de ella, el foco de atención de nuestro grupo de trabajo, se volvió hacia los países hispanoamericanos. El despliegue de actividades académicas y eventos a que fueron invitados sus especialistas, ha favorecido los intercambios y la visibilidad internacional de la psicología cubana en general y de la psicología aplicada a la salud de los trabajadores. 

independiente de la educación superior, y sus egresados la condición de profesionales psicólogos titulados. Hasta entonces la psicología se estudiaba en Bachillerato, y en carreras como Filosofía, Pedagogía y Medicina en calidad de na asignatura más del programa de estudios. Algunos de los egresados ofrecían servicios de orientación, estudios de mercado, diagnóstico clínico, o realizaban labores docentes (Rodríguez, 1990). Solo a fines de la década de los años 50 del pasado siglo, se creó una escuela de psicología en una universidad privada, que desapareció al decretarse la intervención del Estado en la enseñanza. escuelas fundamentales estaban todas representadas. Su propósito era ofrecer las alternativas posibles al estudiante de modo tal que juzgara por sí mismo y no se coactara su libertad de elección y afiliación a uno u otro paradigma teórico. Múltiples autores convergieron en aquel plan de estudios: Freud, James, Wallon, Rubinstein, Allport, Fraisse, Piaget, Luria, Wundt, Vygotsky, Zazzó, Rogers, entre tantos otros. 
Así lo reconoció Fernando González quien años después fuera Decano de la Facultad de Psicología en la Universidad de La Habana :

“...ese eclecticismo fue saludable porque permitió a los estudiantes superar desde un principio el dogmatismo estrecho basado en las teorías psicológicas clásicas..." (González \& Pavón-Cuéllar, 2019, p. 24).

Esa estrategia docente transmitía una idea más real del estado de la disciplina, en que coexistían tendencias y escuelas diferentes, pero no siempre los estudiantes estaban en condiciones de asumir criterios propios.

El proceso de depuración ideológica iniciado en 1968 que se denominó ofensiva revolucionaria, condujo a decisiones radicales. Por contener pasajes críticos a la Unión Soviética, se dio orden de retirar todos los ejemplares del tratado de personalidad de Allport, acabado de editar, así como una compilación de artículos de psicología social realizada por el jefe de departamento de Psicología social, quien fuera uno de los fundadores de la Escuela (luego Facultad) y fue retirado de su cargo. Una edición cubana de las Obras escogidas de Freud corrió la misma suerte a causa de los estudios culturales que componían el tercer tomo.

Al entrar Cuba como miembro pleno de la comunidad socialista, se abrieron amplias oportunidades para la formación postgraduada en los países que la componían, se ampliaron los intercambios institucionales, se descubrieron autores más recientes; pero se perdió la diversidad de las fuentes bibliográficas. En su lugar se importó en bloque la literatura de diversas especialidades y se proclamó la orientación históricocultural como enfoque fundamental.

La obra de Vygotsky, que pretendió construir un enfoque de la psicología desde los principios del materialismo dialéctico, se truncó por su temprana muerte. El enfoque histórico-cultural tuvo un desigual desarrollo en los diversos campos de aplicación de la disciplina. El trabajo y la salud no fueron de los más favorecidos.

En Cuba, los trabajos de elaboración teórica en el campo de la salud ocupacional son escasos; pero fundados en el enfoque histórico-cultural son prácticamente inexistentes.

El psicólogo cubano Fernando González, que dedicó gran parte de su fructífera trayectoria profesional al estudio de la subjetividad ha afirmado rotundamente: "La subjetividad no ha sido un tema importante para la psicología cubana" (González \& Pavón-Cuéllar, 2019, p 23).

Me anticipo a asumir la responsabilidad por no haber dado continuidad a mi trabajo de tesis doctoral en que se propuso y validó un modelo de factores psicosociales laborales en relación con la salud centrado en la subjetividad (Román, 1990). La afluencia de información sobre los modelos de estrés de Karasek y de Siegrist entre otros, y el interés por conocer las recientes formulaciones teóricas sobre el estrés, desvió mi atención y mis esfuerzos en momentos en que la comunicación con nuestros interlocutores exigía conocer a profundidad esas realizaciones.

110 Es preciso reformular los conceptos, inclusive los más conocidos si pretendemos un enfoque coherente desde la subjetividad. De ese modo, pongamos por caso, el riesgo ya no sería un número que exprese una probabilidad estadística, ni un objeto ajeno a nuestras atribuciones; sino precisamente nuestra representación del objeto y de su probable peligro, hasta si se quiere con una noción apreciativa de su probabilidad, y 
todo ello con el añadido de nuestras atribuciones de sentido respecto a cualidades que lo hacen a nuestros ojos frágil, importante, evocador de recuerdos u otras atribuciones.

Dos fuentes más aportadas por profesionales europeos, ideológica y éticamente comprometidos con la clase trabajadora, se sumó al conjunto de referentes con que se iniciaron nuestros trabajos sobre los determinantes, sociales, psicosociales e individuales de la salud de los trabajadores. Primero, el Modelo Obrero Italiano, liderado por Ivar Odonne que destacaba el rol anti-hegemónico del investigador y la sabiduría obrera forjada en el propio trabajo. Un enfoque no ajeno a la formación y práctica de un psicólogo cubano. Segundo, un ejemplar de "Trabajo y desgaste mental", del profesor Christophe Dejours que solidariamente nos llegó en su edición argentina de 1990. Pasaron alrededor de 20 años para que de igual modo recibiéramos "El factor humano" y otras obras más. En lo personal, solo los códigos psicoanalíticos me impiden una mejor comprensión y sacar más provecho de su lectura. De cualquier modo, la identificación con su concepto de la experiencia subjetiva como objeto de la psicología y sus críticas a la teoría del estrés son criterios con que me identifico plenamente.

Bajo la exploración conjunta, acompañada, de la subjetividad de los propios trabajadores podremos descubrir riesgos psicosociales laborales que habíamos pasado por alto porque siendo propios de nuestras condiciones concretas no los habíamos advertido, o tal vez habiendo pretendido colocar el molde de uno u otro modelo reconocido.

113 En esa búsqueda ya iniciada, aparecerán productos autóctonos. Como un riesgo psicosocial las incertidumbres de trabajadores cubanos, contradictoriamente en una sociedad que les ofrece garantías fundamentales; así como la manifiesta e inesperada reacción de satisfacción de no pocos trabajadores jóvenes ante la pérdida de un empleo estatal, estable y seguro a cambio de un posible empleo privado, lo que en otras coordenadas geográficas y sociales probablemente sería un motivo de severa angustia (Román, 2019).

\subsection{Salud mental de los trabajadores}

Los problemas que atañen a la salud mental de los trabajadores son de diverso carácter :

- El trabajo como determinante de la calidad del estado de la salud mental y de trastornos ocasionados por el trabajo.

- Relaciones de la salud mental con alteraciones de la salud física.

- Las competencias de los profesionales de la salud mental para la atención de los trabajadores.

- El reconocimiento de las alteraciones de la salud mental de los trabajadores.

115 El trabajo como factor de desgaste puede ser también fuente de realización y crecimiento personal y colectivo, y modificar la calidad de la salud mental, la que alude a los juicios que cabe formularse en relación con dimensiones tales como funcionalidaddisfuncionalidad, sano-patológico, bienestar-malestar y similares.

116 Los estados de malestar relacionados con el trabajo han permitido describir alteraciones, como el burnout, karoshi, estrés laboral, acoso psicológico. Su especificidad, así como sus relaciones con alteraciones somáticas reclaman una formación especial de los profesionales. 
117 No pocas investigaciones han permitido observar asociaciones entre ciertos trastornos y enfermedades somáticas, como la hipertensión arterial y la diabetes con condiciones psicosociales laborales.

118 Las alteraciones de la salud mental vinculadas al trabajo no han recibido en todos los países su reconocimiento en calidad de trastorno psiquiátrico. Reconocerlas además como enfermedades profesionales ha dado lugar a extensos debates para demostrar su exclusivo origen laboral (Organización Internacional del Trabajo, 2010).

119 En la última revisión técnica de la lista, que entrañó un largo proceso de múltiples consultas, la OIT sacó a debate la posibilidad de incorporar un acápite dedicado a las alteraciones mentales; sin modificar el requisito del origen laboral.

Además, propuso considerar tres alteraciones: depresión, trastorno por estrés postraumático y síndrome psiquiátrico por efecto del acoso laboral.

La lista finalmente aprobada como recomendación a los países miembros incluyó el controvertido acápite, pero solamente con el trastorno por estrés postraumático. Cuba, al revisar su lista, adoptó una decisión tan tajante como conservadora : no incluyó el acápite de alteraciones mentales, ni ninguna de ellas.

Los profesionales de la salud mental cubanos psiquiatras, psicólogos, enfermeros y tecnólogos de la salud necesitarían de una formación complementaria para enfrentar correctamente las demandas de atención de la salud mental de los trabajadores. De otro modo no estarían al nivel profesional y la calidad de los servicios que en el reputado sistema nacional de salud cubano poseen otras especialidades. Existen condiciones para hacerlo.

\subsection{Consideración final}

123 Espero haber podido transmitir una fundamentada preocupación por el empobrecimiento progresivo de la salud ocupacional cubana, al punto de verse amenazada su propia existencia ; o lo que es prácticamente lo mismo, una involución hacia un practicismo extremo, manifestación de mediocridad científica, lo que conduce a una limitada capacidad de dar respuestas eficaces, de validez y confiabilidad comprobables, a las necesidades que en este campo tiene y tendrá el país.

124 Confío en que la psicología tendrá un mejor destino. El trabajo de largos años ha creado las condiciones a nuestro alcance para que sea así. La psicología de la salud ocupacional en Cuba ha rebasado los marcos institucionales en que surgió.

125 Hoy prácticamente no es concebible un programa de psicología del trabajo o de psicología de la salud en el país que no incluya su intersecto como objeto de conocimiento propio. Cada vez, más profesionales desde sus respectivas instituciones trabajan en este campo, e inclusive dedican a él sus trabajos de tesis de maestrías y de doctorado. En ellos también reside la garantía de la continuidad de la psicología de la salud ocupacional cubana, 


\section{Conclusiones}

Los análisis realizados permiten formular los juicios conclusivos siguientes :

a. Es lamentablemente paradójico que la Constitución cubana defina al país como un Estado de trabajadores y que la Salud Ocupacional y las especialidades que conforman esa multidisciplina, incluida la psicología, se encuentren en situación crítica.

b. La relación entre las demandas actuales y previsibles a la salud ocupacional, por una parte, y los recursos materiales, humanos y organizativos, por la otra resulta desventajosa, por cuanto las primeras superan a los segundos.

c. La clausura de la residencia de Medicina del trabajo limita en extremo la función social de la salud ocupacional, e inclusive hace peligrar su existencia, lo cual repercutiría negativamente sobre la psicología y las restantes especialidades que convergen en ese espacio multidisciplinario.

d. El diálogo entre la salud ocupacional y las ciencias sociales, así como con otras especialidades médicas es aún limitado, a pesar de que ha de resultar mutuamente productivo y útil.

e. La aplicación de la ciencia psicológica a la salud ocupacional se ha caracterizado por la sistematicidad de sus resultados en las investigaciones y la docencia, principalmente.

f. El positivismo es aún el enfoque prevalente en las investigaciones y la práctica profesional, aunque se han iniciado trabajos con una perspectiva psicodinámica basadas en la subjetividad, lo que requiere una reformulación de sistema conceptual tradicional de la salud ocupacional.

g. El enfoque histórico-cultural de L.S. Vygotsky es la referencia teórica fundamental de los psicólogos cubanos en el problema de la subjetividad.

h. La psicología de la salud ocupacional cubana puede basar sus perspectivas no solo en los profesionales del sistema nacional de salud ; sino además en otros pertenecientes a sectores, instituciones académicas y organizaciones laborales que, habiendo recibido una formación complementaria, desarrollan trabajos de esa especialidad.

\section{BIBLIOGRAFÍA}

Almirall, P. (2001). Neurotoxicología. Apuntes teóricos y aplicaciones prácticas. Caracas : Imprenta universitaria de la Universidad Central de Venezuela.

Almirall, P., Mayor, J., Del Castillo, N., Rodríguez, R., \& Román, J. (1987). Manual de recomendaciones para la evaluación psicológica de trabajadores expuestos a sustancias neurotóxicas. La Habana : Instituto de Medicina del Trabajo.

Asamblea Nacional del Poder Popular (ANPP) (1977). Ley 13 de Protección e Higiene del Trabajo. La Habana : Consultado en : www.aclifim.sld.cu/Leyes/Ley13.htm

Asamblea Nacional del Poder Popular (ANPP) (2014). Ley 116/2013. Gaceta Oficial de la República de Cuba, № 29, Año CXII [Extraordinaria], 17 de junio de 2014. Consultado en : http://

www.gacetaoficial.gob.cu 
Barral, F. (2010). Mis vidas sucesivas. Recuerdos y destino de un niño de la guerra. La Habana : Ediciones "La Memoria". Centro Cultural "Pablo de la Torriente Brau".

Carrión, M. A., López, F., \& Tous, J., (Eds.). (2007). Evaluación e intervención psicosocial. Barcelona : Asociación de expertos en psicosociología aplicada.

Constitución de la República de Cuba (2019, 10 de abril). Gaceta Oficial de la República de Cuba, № 5, Año CXVII [Extraordinaria]. Consultado en : http://www.gacetaoficial.gob.cu

Dejours, C. (1990). El desgaste mental en el trabajo. Buenos Aires : Humanitas (2 $2^{a}$ edición).

Figueredo, O., \& Concepción, J. R. (2018). Economía cubana : Balance de 2018 y perspectivas para el futuro inmediato. Consultado en : http://www.cubahora.cu/economia/economia-cubana-balancede-2018-y-perspectivas-para-el-futuro-inmediato

García, E., \& Granda, A. (2013). Proposición para perfeccionar la conquista social de la seguridad y salud de los trabajadores en Cuba. Revista Cubana de Salud Pública, 39(3) 19-23.

González, F., \& Pavón-Cuéllar, D. (2019). La revolución cubana : subjetividad y psicología. Teoría y crítica de la psicología. 12(1),10-35, http://www.teocripsi.com/ojs/

Granda, A. (1992). La Salud Ocupacional en Cuba. Revista Médica del Uruguay. 8(3), 13-14.

Medina, A. (2018). Programa de formación de competencias para la gestión eficaz de la seguridad y salud en el trabajo. Evaluación en una empresa biotecnológica. (Tesis de Doctorado). Facultad de Psicología, Universidad de La Habana, La Habana, Cuba.

Ministerio de Salud Pública (MINSAP) (1995-2018). Anuarios Estadísticos de Salud 1995-2018. La Habana : Consultado en : http://files.sld.cu/dne/files/

Ministerio de Salud Pública (MINSAP) (1986-2002). Informes anuales de la Dirección de Registros Médicos y Estadísticas de Salud 1986 a 2002. La Habana.

Molina, E. (2016). Devenir del modelo económico socialista. La Habana : Editorial de Ciencias Sociales. Oficina Nacional de Estadística e Información (ONEI) (2019) Anuario estadístico de Cuba. La Habana. Organización Internacional del Trabajo (OIT) (2010). Lista de enfermedades profesionales (revisada en 2010). Identificación y reconocimiento de las enfermedades profesionales : Criterios para incluir enfermedades en la lista de enfermedades profesionales de la OIT. Serie Seguridad y Salud en el Trabajo. Ginebra : Oficina Internacional del Trabajo, (74).

Organización Mundial de la Salud (OMS) (1974). Actas oficiales de la Organización Mundial de la Salud, № 217. Ginebra : Asamblea Mundial de la Salud, 7-23 de mayo. Parte 1.

Orozco, O., Del Castillo, N., \& Román, J., Eds. (2018). Seguridad integral en el trabajo. Un enfoque psicosocial. Cali : Editorial Bonaventuriana.

Rodríguez, A. (1990). Transitando por la psicología. Antes y después de la revolución. La Habana : Editorial de Ciencias Sociales.

Román, J. (1990). Los factores psicosociales y la salud del trabajador : un procedimiento para su evaluación (Tesis de Doctorado). Facultad de Psicología. Universidad de La Habana, La Habana, Cuba.

Román, J. (2017a). El especialista en salud mental en Cuba ante la actividad laboral, Alternativas cubanas en Psicología, 5(14), 12-27.

Román, J. (2017b). La salud ocupacional en Cuba. Pasado, presente y perspectivas futuras. Anales de la Academia de Ciencias de Cuba. 7(3). 
Román, J. (2019). Riesgos, trabajo y sociedad : la subjetividad como vínculo. Teoría y Crítica de la Psicología. 12, 97-116. Consultado en : teocripsi.com/ojs/index.php/TCP/article/view/307

Universidad de Ciencias Médicas de La Habana (2016). Plan de estudio de la carrera de medicina. Documento consultado en la Dirección de Pregrado (15-12-2018).

Universidad de Ciencias Médicas de La Habana (2016). Plan de estudio de la residencia médica de higiene y epidemiología. Documento consultado en el Departamento de Docencia del Instituto Nacional de Higiene, Epidemiología y Microbiología (15-12-2018).

Universidad de Ciencias Médicas de La Habana (2016). Plan de estudio de la residencia médica de Psiquiatría. Documento consultado en el Departamento de Docencia de la Facultad de Ciencias Médicas "Calixto García” (16-12-2018).

Universidad de Ciencias Tecnológicas. Ingeniería industrial (2016). Plan de estudios D. Consultado en : http://cujae.edu.cu/industrial/carrera/ingeniería-industrial

Varillas, W, Ed. (2009). Los trabajadores y la salud mental. El reto de la prevención y el tratamiento en un mundo enfermo. La Habana : Editorial Ciencias Médicas.

\section{RESÚMENES}

El artículo tiene como objetivo comunicar el origen y la evolución de la aplicación de la ciencia psicológica al campo multidisciplinario de la salud ocupacional en Cuba. Se tienen en cuenta diversos factores en este proceso: el contexto social y socio-laboral, con una exposición relativamente extensa dada la singularidad del sistema social cubano. La trayectoria de la salud ocupacional en el país y su situación actual crítica. Las aportaciones de la práctica profesional de los psicólogos de la salud en los escenarios laborales que dieron origen a la psicología de la salud ocupacional como una línea de trabajo en la investigación, la docencia y la práctica profesional. Se enfatiza la necesidad de desarrollar trabajos teóricos y metodológicos en esa área desde un punto de vista psicodinámico, que tenga en cuenta la subjetividad, para la que se propone el enfoque histórico cultural de L. S. Vygotsky.

O artigo tem como objetivo comunicar a origem e a evolução da aplicação da ciência psicológica ao campo multidisciplinar da saúde ocupacional em Cuba. Vários fatores são levados em consideração nesse processo: O contexto social e sócio-laboral, com uma exposição relativamente extensa, dada a singularidade do sistema social cubano. A trajetória da saúde ocupacional no país e sua situação atual crítica. As contribuições da prática profissional dos psicólogos em cenários de trabalho, que deram origem à psicologia da saúde ocupacional como uma linha de trabalho em pesquisa, ensino e prática profissional. Enfatiza a necessidade de desenvolver trabalhos teóricos e metodológicos nessa área do ponto de vista psicodinâmico, que leva em conta a subjetividade, para a qual é proposta a abordagem histórica cultural de L. S. Vygotsky.

L'article veut exposer l'origine et l'évolution de l'application de la science psychologique au domaine multidisciplinaire de la santé au travail à Cuba. Plusieurs facteurs sont pris en compte dans ce processus: le contexte social et socio-professionnel, qui exige un exposé développé compte tenu du caractère singulier du système social cubain; la trajectoire de la question de la santé au travail dans le pays et sa situation critique actuelle; les contributions de la pratique professionnelle des psychologues dans les scénarios de travail, qui sont à la source de la psychologie de la santé au travail en tant que domaine d'activité de recherche, d'enseignement et de pratique professionnelle. On souligne la nécessité de développer des travaux théoriques et 
méthodologiques dans ce domaine, sous un point de vue psychodynamique qui prenne en compte la subjectivité. L'approche culturelle et historique de L. S. Vygotsky est alors proposée.

The paper aims at communicating the origin and evolution of the application of psychological science to the multidisciplinary field of occupational health in Cuba. Several factors are taken into account in this process: the social and socio-labor context, with a relatively extensive exposure, given the uniqueness of the Cuban social system. The trajectory of occupational health in the country and its current critical situation. The contributions of the professional practice of psychologists in work scenarios, which gave rise to occupational health psychology as a line of work in research, teaching and professional practice. It emphasizes the need to develop theoretical and methodological work in this area from a psychodynamic point of view, which takes subjectivity into account, for which the cultural historical approach of L. S. Vygotsky is proposed.

\section{ÍNDICE}

Keywords: occupational health, occupational health psychology, subjectivity, Vygotski, Cuba Palabras claves: salud ocupacional, psicología de la salud ocupacional, subjetividad, Vygotsky, Cuba

Palavras-chave: saúde ocupacional, psicologia da saúde ocupacional, subjetividade, Vygotsky, Cuba

Mots-clés: santé au travail, psychologie de la santé au travail, subjectivité, Vygotsky, Cuba

\section{AUTOR}

\section{JORGE JUAN ROMÁN HERNÁNDEZ}

Universidad de Ciencias Médicas de La Habana. Facultad de Ciencias Médicas “Julio Trigo". Calzada de Bejucal, 7.5 km, CP 10900, La Habana, Cuba, roman@infomed.sld.cu 